\title{
MicroRNA-375 plays a dual role in prostate carcinogenesis
}

\author{
Pedro Costa-Pinheiro ${ }^{1}$, João Ramalho-Carvalho ${ }^{1 \dagger}$, Filipa Quintela Vieira ${ }^{1,2+}{ }^{+}$, Jorge Torres-Ferreira ${ }^{1}$, Jorge Oliveira ${ }^{3}$,
} Céline S Gonçalves ${ }^{5,6}$, Bruno M Costa ${ }^{5,6}$, Rui Henrique ${ }^{1,4,7}$ and Carmen Jerónimo ${ }^{1,7^{*}+}$

\begin{abstract}
Background: Prostate cancer (PCa), a highly incident and heterogeneous malignancy, mostly affects men from developed countries. Increased knowledge of the biological mechanisms underlying PCa onset and progression are critical for improved clinical management. MicroRNAs (miRNAs) deregulation is common in human cancers, and understanding how it impacts in PCa is of major importance. MiRNAs are mostly downregulated in cancer, although some are overexpressed, playing a critical role in tumor initiation and progression. We aimed to identify miRNAs overexpressed in PCa and subsequently determine its impact in tumorigenesis.

Results: MicroRNA expression profiling in primary PCa and morphological normal prostate (MNPT) tissues identified 17 miRNAs significantly overexpressed in PCa. Expression of three miRNAs, not previously associated with PCa, was subsequently assessed in large independent sets of primary tumors, in which miR-182 and miR-375 were validated, but not miR-32. Significantly higher expression levels of miR-375 were depicted in patients with higher Gleason score and more advanced pathological stage, as well as with regional lymph nodes metastases. Forced expression of miR-375 in PC-3 cells, which display the lowest miR-375 levels among PCa cell lines, increased apoptosis and reduced invasion ability and cell viability. Intriguingly, in 22Rv1 cells, which displayed the highest miR-375 expression, knockdown experiments also attenuated the malignant phenotype. Gene ontology analysis implicated miR-375 in several key pathways deregulated in PCa, including cell cycle and cell differentiation. Moreover, CCND2 was identified as putative miR-375 target in PCa, confirmed by luciferase assay.
\end{abstract}

Conclusions: A dual role for miR-375 in prostate cancer progression is suggested, highlighting the importance of cellular context on microRNA targeting.

Keywords: Prostate cancer, MicroRNAs, Epigenetics, miR-375, CCND2

\section{Background}

Prostate cancer $(\mathrm{PCa})$, the second most incident cancer in men worldwide (31.1\%) [1] and ranking first in incidence in the US (27\%) [2], is very heterogeneous, ranging from clinical indolent to extremely aggressive disease, causing substantial morbidity and mortality [3]. Adequate management is, thus, mandatory to avoid overtreatment, on one hand, and sub-optimal therapy,

\footnotetext{
* Correspondence: carmenjeronimo@ipoporto.min-saude.pt

${ }^{\dagger}$ Equal contributors

${ }^{1}$ Cancer Biology and Epigenetics Group - Research Center (Lab3), Portuguese Oncology Institute - Porto (IPO-Porto), Rua Dr. António Bernardino de Almeida, Porto 4200-072, Portugal

${ }^{7}$ Department of Pathology and Molecular Immunology, Institute of Biomedical Sciences Abel Salazar (ICBAS), University of Porto, Rua de Jorge Viterbo Ferreira 228, Porto 4050-313, Portugal

Full list of author information is available at the end of the article
}

on the other. A better understanding of the biological mechanisms underlying $\mathrm{PCa}$ onset and progression are likely to contribute to improved clinical and therapeutic management.

Over the last two decades, deregulation of epigenetic mechanisms has emerged as a relevant driving force in $\mathrm{PCa}$ [4], with recent emphasis in altered microRNAs (miRNAs) expression [5]. MicroRNAs are a class of small non-coding RNAs, approximately 22 nucleotides in length [6], highly conserved along the evolutionary chain, with tissue and developmental stage-specific expression [7]. Currently, more than 2,000 human miRNAs are registered in the miRBase [8] and thought to negatively regulate gene translation, thus decreasing gene expression, and have been extensively implicated in several crucial cellular pathways, such as apoptosis, 
differentiation, and proliferation [9]. Interestingly, a single miRNA might have multiple targets, and a single mRNA may be targeted by several miRNAs [10].

In cancer, miRNAs are globally downregulated, although some are notoriously upregulated [11]. Such trend has been found in $\mathrm{PCa}$, but most studies limit their analysis to expression array results, based on few tumor samples, and generally lacking subsequent validation in larger and independent datasets [5,12]. This may, at the least partially, explain contradictory results in the literature, making it difficult to establish specific PCa miRNA signatures [13]. Thus, assessing miRNAs differential expression in a robust cohort of patients carrying primary tumors and searching for miRNAs targets are critical to investigate its relevance in PCa initiation and progression.

In this study, we sought to discover miRNAs upregulated in PCa and unveil its role in prostate carcinogenesis through modulation of miRNAs expression and identification of putative molecular targets. Using a customized, commercially available, platform, a small set of miRNAs upregulated in $\mathrm{PCa}$ was identified, some of which have been previously reported. Validation in two large independent sets of patients demonstrated that miR-375 expression was increased in PCa with higher Gleason score and more advanced pathological stage, which entail worse prognosis. Following validation in clinical samples, miR-375 was also found to be upregulated in PCa cell lines compared to RWPE-1 (a benign prostate epithelial cell line). Modulation of miR-375 expression in two PCa cell lines (22Rv1 and PC-3) showed that this miRNA is involved in regulation of cell viability and apoptosis, in a cell-context-dependent manner. Furthermore, using a custom gene panel to search for potential targets followed by specific luciferase assay validation, CCND2 was identified and confirmed as miR-375 target in PCa. Our observations thus suggest that miR-375 overexpression may contribute to prostate carcinogenesis and disease progression.

\section{Results}

\section{MicroRNAs expression in prostate cancer tissues}

Global expression of miRNAs was initially assessed and compared in ten $\mathrm{PCa}$ and four morphological normal prostate (MNPT) samples. Global miRNAs downregulation was found in $\mathrm{PCa}$, although overexpression, with fold variation higher than 1.5, was depicted for 17 miRNAs (Table 1). Among these, those previously associated with $\mathrm{PCa}$ in the literature were excluded from further analysis, and the remainders were further selected for validation in a large sample set, and the respective clinical and pathological characteristics are provided in Table 2 (Additional file 1: Table S1). No significant differences in age were apparent between the two groups. Because several miRNAs were below detection level in quantitative reverse transcriptionpolymerase chain reaction (RT-qPCR) analyses (probably
Table 1 List of overexpressed microRNAs in low-density miRNA RT-qPCR analysis (fold variation represents median values for PCa vs. MNPT)

\begin{tabular}{ll}
\hline MicroRNA & Fold variation (PCa vs. MNPT) \\
\hline miR-449a\# & 3.92 \\
miR-32 & 3.49 \\
miR-548c-5p & 2.71 \\
miR-562 & 2.56 \\
miR-103-as & 2.53 \\
miR-512-3p & 2.41 \\
miR-200c* & 2.33 \\
miR-147b & 2.24 \\
miR-770-5p & 2.09 \\
miR-518c* & 2.00 \\
miR-517b & 1.88 \\
miR-182 & 1.79 \\
miR-615-3p & 1.70 \\
miR-496 & 1.59 \\
miR-1200 & 1.58 \\
miR-375 & 1.54 \\
miR-551a & 1.53 \\
\hline
\end{tabular}

*Passanger strand. \#Already studied in other work by our research group. MNPT, morphological normal prostate; PCa, prostate cancer. In italics are the microRNAs chosen for further validation.

due to low expression levels), only three miRNAs (miR-32, miR-182, and miR-375) were assessed in the larger dataset. Whereas miR-182 and miR-375 were significantly overexpressed in $\mathrm{PCa}(P<0.001$ for both), confirming the results of the array, no significant differences were found for miR-32 expression between $\mathrm{PCa}$ and MNPT (Figure 1A and Additional file 2: Figure S1). MiR-375 expression levels were significantly higher in cases with higher Gleason score and more advanced pathological stage at diagnosis $(P<0.05, P<0.001$, Figure $1 \mathrm{~B}, \mathrm{C})$.

Table 2 Clinical and pathological data of patients included in miR-375 validation

\begin{tabular}{lll}
\hline Clinicopathological data & Tumors $(\boldsymbol{n}=1 \mathbf{1 4})$ & MNPT $(\boldsymbol{n}=\mathbf{1 5})$ \\
\hline Age (years), median (range) & $65(49$ to 74$)$ & $64(45$ to 80$)$ \\
PSA (ng/mL), median (range) & $8.00(2.66$ to 20.00$)$ & n.a. \\
Pathological stage, $n(\%)$ & & \\
pT2 & $58(38.7 \%)$ & n.a. \\
pT3a & $24(16.0 \%)$ & n.a. \\
pT3b & $33(21.3 \%)$ & n.a. \\
Gleason score, $n(\%)$ & & \\
$<7$ & $28(18.7 \%)$ & n.a. \\
$=7$ & $73(48.7 \%)$ & n.a. \\
$>7$ & $13(8.7 \%)$ & n.a. \\
\hline
\end{tabular}

n.a., not applicable; MNPT, morphological normal prostate. 


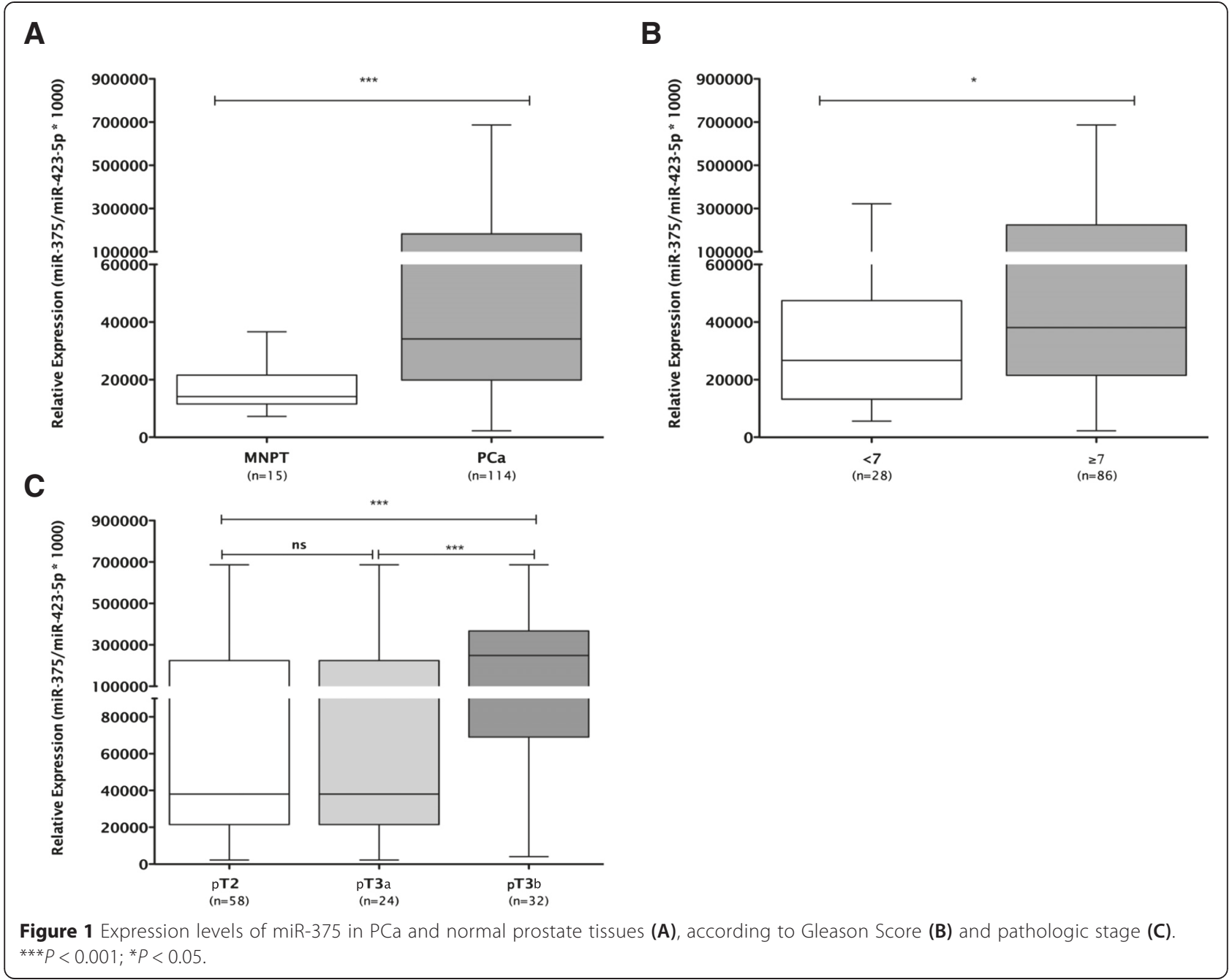

\section{Meta-analysis of miRNA expression in prostate cancer patients from The Cancer Genome Atlas}

Further validation of the array results was performed in a larger and independent dataset, that is, the miRNAseq expression data from PCa patients and matched normal samples deposited in The Cancer Genome Atlas (TCGA) ( $n=326$ and $n=50$, respectively). Strikingly, miR-375 was significantly overexpressed in all tumors compared to matched normal samples $(P<0.0001$; Figure $2 \mathrm{~A})$, as well as in patients with regional lymph node metastasis (N1) compared to those without regional lymph node involvement (N0) $(P=0.0017$; Figure $2 \mathrm{~B})$. Moreover, both miR-32 and miR-182 were overexpressed in PCa compared to matched normal prostate tissues $(P<0.0001$; Additional file 3: Figure S4).

Correlation analysis for miRNAs expression showed that miR-375 was significantly co-expressed with miR-32 and miR-182 ( $r=0.36$ and $r=0.60$, respectively; Table 3 and Figure 3).

\section{MiR-375 expression in prostate cell lines}

PCa cell line 22Rv1 depicted the highest miR-375 expression levels, whereas the lowest were found in PC-3 cells (Figure 4). In RWPE-1 cells, miR-375 expression levels were lower than those of any PCa cell line, mimicking the results of PCa and MNPT tissues. Thus, those cell lines were selected for subsequent functional experiments of miR-375 downregulation (22Rv1) or forced expression (PC-3 and RPWE-1).

Phenotypic impact of miR-375 forced expression in PC-3 cells and RWPE- 1 cells

At $72 \mathrm{~h}$ after transfection, miR-375 expression levels were increased 56,000 and 8,000 times in PC-3 and RPWE-1 cells, respectively ( $P<0.001$ for both) (Figures $5 \mathrm{~A}$ and $6 \mathrm{~A}$ ). PC-3 cells' viability was significantly reduced at $48 \mathrm{~h}$ (39\%, $P<0.01)$ and $72 \mathrm{~h}(60.5 \%, P<0.001)$ (Figure 5B). Moreover, $72 \mathrm{~h}$ after transfection, apoptosis levels were significantly increased (more than threefold $(P<0.001)$, Figure 5C). 


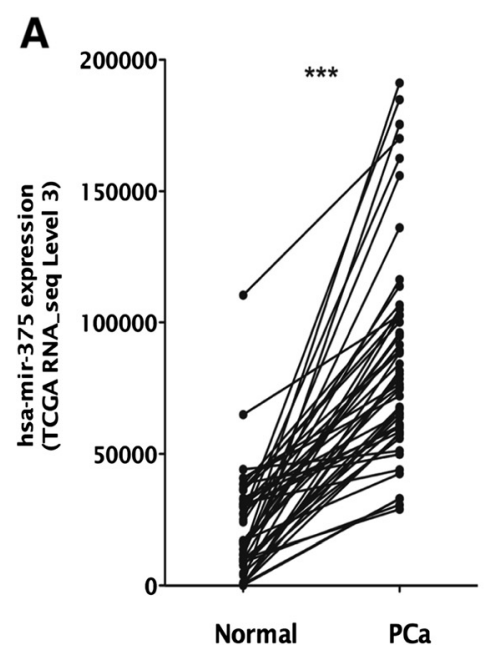

B

Figure 2 Expression of has-mir-375 is increased in prostate cancer and associates with lymph node stage in patients from TCGA. (A) miR-375
expression (TCGA miRNAseq RPKM level 3 value) was evaluated in 50 tumor and matched normal samples of prostate adenocarcinoma patients from
TCGA. All tumor samples presented overexpression comparing to their matched normal samples (***P<0.0001). (B) The expression of hsa-mir-375
(TCGA miRNAseq RPKM level 3 value) was assessed in 177 prostate adenocarcinoma patients from TCGA with lymph node stage information available.
Patients in the N1 lymph node stage presented significantly higher expression of hsa-mir-375 than patients in the N0 stage.

Because PC-3 is highly invasive, this feature was evaluated following miR-375 forced expression and a maximum of $52 \%$ reduction was observed in transfected cells $(P<0.01)$ (Figure 5D and Additional file 4: Figure S2). In transfected RWPE-1 cells, and despite a significant increase in miR-375

Table 3 Validation of the correlation between hsa-mir-375 and identified upregulated microRNAs in prostate adenocarcinoma samples from TCGA dataset [40]

\begin{tabular}{lll}
\hline microRNA & Spearman's correlation & $P$ value \\
\hline hsa-mir-449a & 0.27351 & $<0.0001$ \\
hsa-mir-32 & 0.36431 & $<0.0001$ \\
hsa-mir-548C & N/A & \\
hsa-mir-562 & N/A & \\
hsa-mir-103-1-AS & N/A & \\
hsa-mir-103-2-as & N/A & \\
hsa-mir-512-1 & 0.13177 & 0.01729 \\
hsa-mir-512-2 & 0.05064 & 0.36211 \\
hsa-mir-200C & 0.59926 & $<0.0001$ \\
hsa-mir-147B & 0.12526 & 0.02370 \\
hsa-mir-770 & -0.09100 & 0.10099 \\
hsa-mir-518C & -0.01563 & 0.77866 \\
hsa-mir-517B & -0.03261 & 0.55740 \\
hsa-mir-182 & 0.60472 & $<0.0001$ \\
hsa-mir-615 & 0.39231 & $<0.0001$ \\
hsa-mir-496 & -0.23667 & $<0.0001$ \\
hsa-mir-1200 & N/A & \\
hsa-mir-551a & -0.00138 & 0.98014 \\
\hline N/A, not applcable & &
\end{tabular}

$\mathrm{N} / \mathrm{A}$, not applicable. levels, no significant alterations in cell viability and apoptosis were apparent (Figure 6B, C), suggesting that miR-375 deregulation is mostly relevant in the context of malignant prostate cell.

\section{Phenotypic impact of miR-375 downregulation in 22Rv1 cells}

Expression levels of miR-375 were decreased by $68 \% 72 \mathrm{~h}$ after transfection $(P<0.001$; Figure $7 \mathrm{~A})$ and this was associated with significantly reduced cell viability $(P<0.01)$. At this time point, viable cells reached only $17 \%$ (Figure $7 \mathrm{~B}$ ) and apoptosis increased by $30 \%(P<0.01)$ in $22 \mathrm{Rv} 1-$ transfected cells (Figure 7C). Because this cell line displays low invasive ability, invasion assay was not performed.

\section{Putative miR-375 targets}

The search for putative miR-375 targets comprised expression analysis of 61 genes implicated in some of the most important cellular pathways deregulated in cancer. Thus, expression profiles of PC-3 transfected (pre-miR-375) and 22Rv1 transfected (anti-miR-375) cells were compared to its respective controls (Additional file 5: Figure S3). In 22Rv1 cell with miR-375 downregulation, $R B 1$ was upregulated, whereas in miR-375-overexpressing PC-3 cells, CCND2 was downregulated $(P<0.001$ for both). These findings were validated in tissue samples as $R B 1$ transcript levels were significantly increase and CCND2 expression levels were significantly decreased in primary $\mathrm{PCa}$ compared to MNPT (Figure 8). Furthermore, gene ontology enrichment analysis disclosed that genes involved in cell cycle regulation were those more frequently deregulated in 

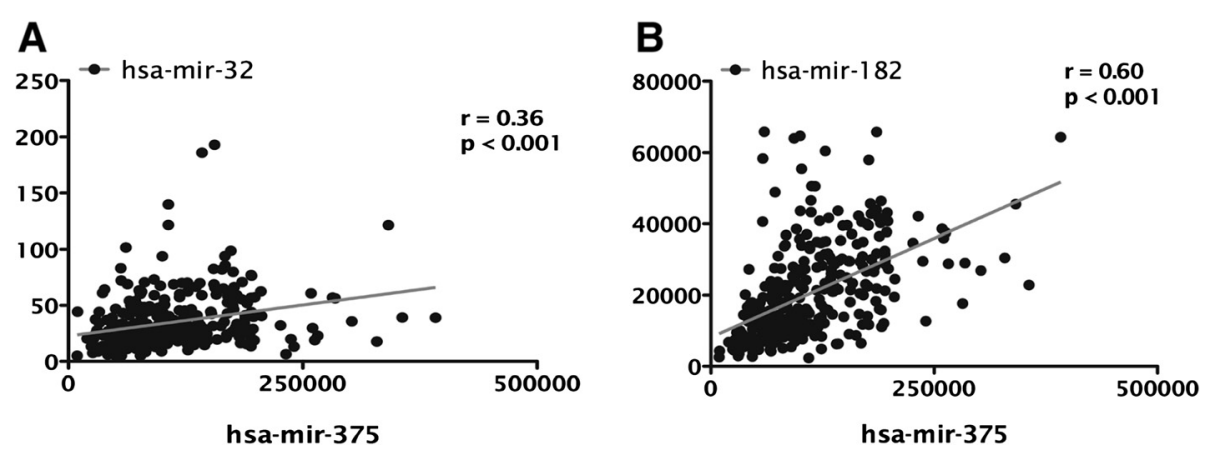

Figure 3 miR-375 is significantly co-expressed with (A) miR-32 and (B) miR-182 in prostate cancer patients from TCGA ( $r$ calculated by Spearman's correlations).

22Rv1 and PC-3 cell lines (Figure 9), supporting a key role for miR-375 in PCa.

\section{miR-375 directly targets CCND2}

In silico analysis identified a miR-375 potential binding site at CCND2 3'untranslated region (UTR). Moreover, analysis of TCGA data disclosed a statistically significant negative correlation between miR-375 and CCND2 expression in PCa tissues (Spearman's correlation, $r=-0.57, P<0.0001$ ). Luciferase assay was performed in PC-3 cells to determine whether miR-375 might regulate CCND2 transcription levels. In CCND2 3'UTR vector and pre-miR-375 cotransfected PC-3 cells, a sixfold increase in miR-375 expression levels was apparent at $72 \mathrm{~h}$ (Figure 10A), whereas CCND2 3'UTR luciferase activity was $37 \%$ reduced, at $48 \mathrm{~h}$ $(P<0.01)$, and $71 \%$ at $72 \mathrm{~h}(P<0.001)$ after transfection (Figure 10B).

\section{Discussion}

Prostate cancer remains a major challenge, mostly due to insufficient knowledge about the factors determining

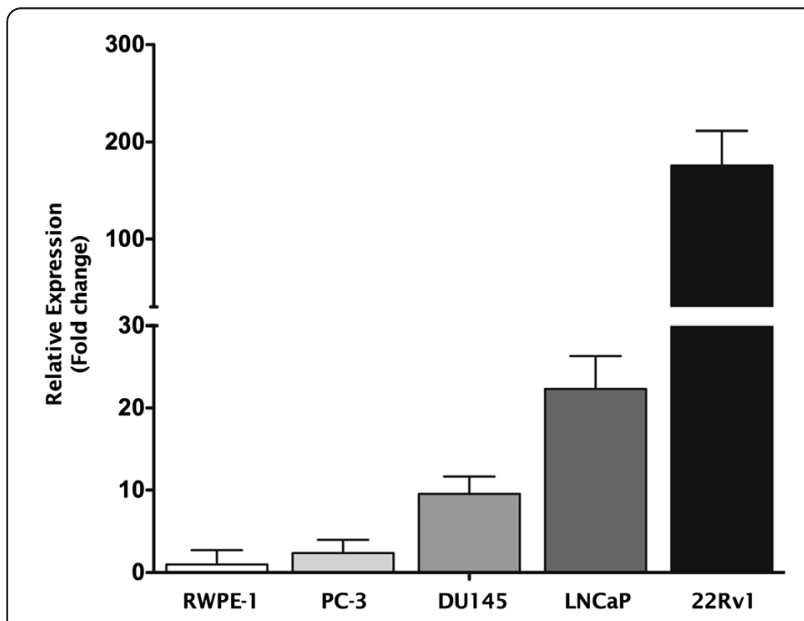

Figure 4 miR-375 expression levels in prostate cell lines. Results are displayed after normalization to RWPE-1. its onset and progression [14]. Because epigenetic alterations play an important role in prostate carcinogenesis and owing to the relative shortage of validated data on miRNA altered expression in PCa, we aimed to identify and validate miRNAs upregulated in this malignancy. Furthermore, its impact on malignant cell phenotype was assessed and putative target genes were identified. The strategy used in our study is similar to that of some previous publications on this subject $[15,16]$.

Results of the miRNA expression array showed that, in PCa, miRNAs are mostly downregulated, whereas only a minor subset is overexpressed, confirming previous reports [13]. Because expression array data may be biased owing to the (usually) small number of samples assessed [17], we decided to validate the miRs overexpressed in the array, and not previously associated with $\mathrm{PCa}$, in two large and independent datasets. Importantly, miR-182 and miR-375 overexpression was confirmed in the validation datasets. However, miR-32 overexpression was not validated and expression of other miRNAs was found to be minimal or absent. These observations further emphasize the need of validation studies following expression array experiments, as well as possible technical limitations for miRNA analysis.

Because a report on miR-182 overexpression in $\mathrm{PCa}$ was published during the execution of this study [18], we then proceeded with miR-375 for further analysis. Although MiR-375 expression levels in body fluids had been previously proposed as diagnostic and prognostic PCa biomarkers [19-21], its biological role in prostate carcinogenesis has not been investigated before. We found that, in PCa tissues, miR-375 expression was higher than in normal prostate tissues, paralleling the results of body fluids analysis [19-21]. Furthermore, higher expression levels associated with higher Gleason score and more advanced pathological stage, two clinicopathological parameters that entail unfavorable prognosis. These observations were further validated in an independent dataset through meta-analysis of TCGA 


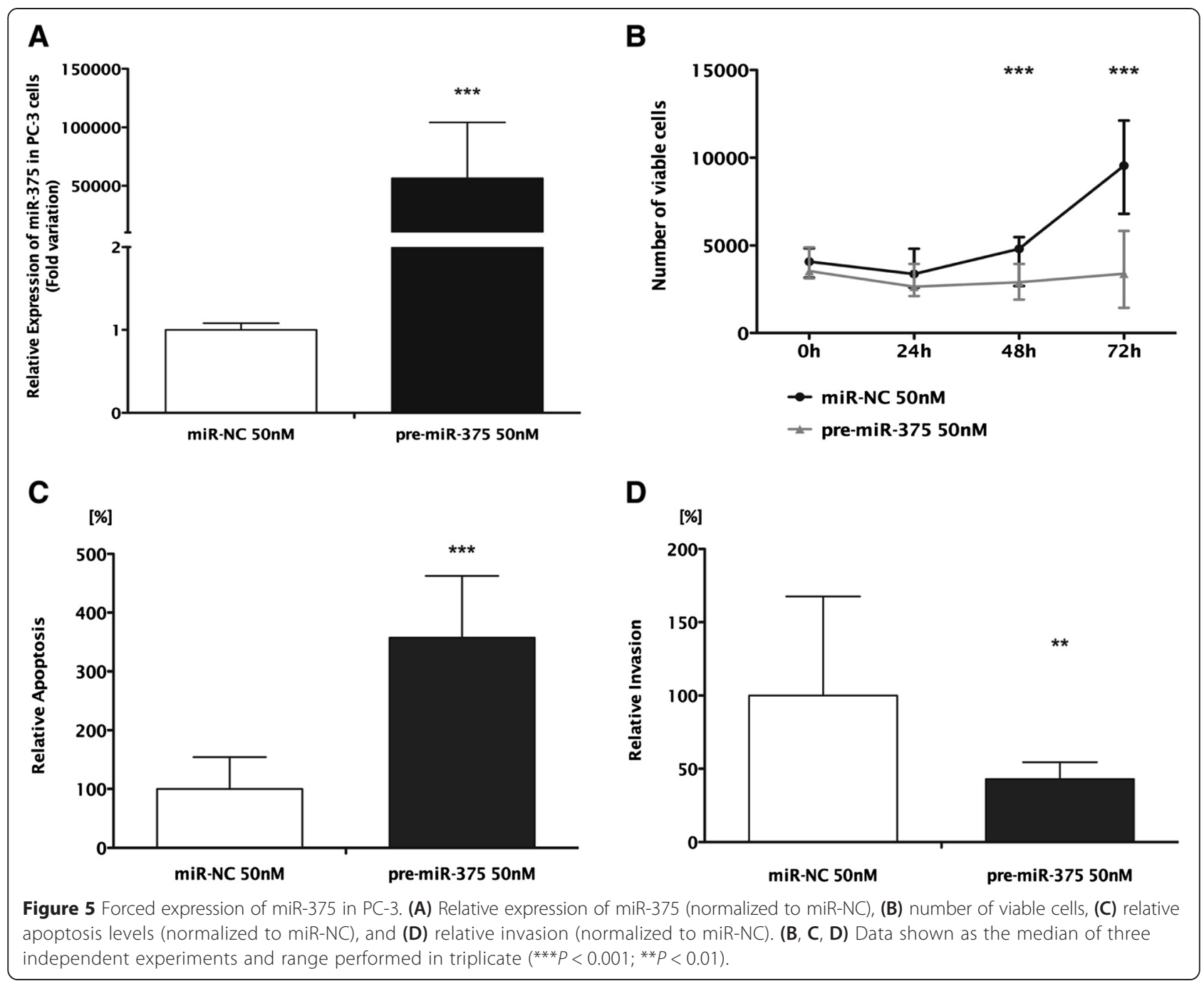

data available for miR-375 expression in prostate tissues. Interestingly, an association of higher miR-375 expression levels and regional lymph node metastases was depicted, providing additional confirmation of our results. Although miR-32 expression levels did not differ between PCa and MNPT in our dataset, the larger number of samples available at the TCGA demonstrated increased expression PCa compared to matched normal prostate tissues. It is noteworthy that normal samples differ not only in number (15 vs. 50) but also in its nature as we used MNPT from patients not carrying $\mathrm{PCa}$ whereas normal prostate tissues from TCGA correspond to matched samples from PCa patients.

Although this and other studies [19-21] implicate miR-375 upregulation in $\mathrm{PCa}, \mathrm{miR}-375$ has been mostly considered a tumor suppressor, namely in gastric, head and neck, pancreatic, and hepatocellular cancers [22-25]. Indeed, in gastric cancer, miR-375 forced expression increased apoptosis and reduced of cell viability in vitro [25], and janus kinase $2(J A K 2)$ was identified as a direct target [26]. It should be emphasized, however, that miR375-altered expression reports are mostly based in microarray or validation platform analysis attempts to discriminate different tumor subgroups according to miRNAs expression, seldom providing biological clues to the role of miR-375 in cancer [19,20,27-29].

Expression analysis results of miR-375 in prostate cells lines parallel those of primary tissues, as the lowest levels were found in RWPE-1, a benign prostate cell line. However, whereas 22Rv1 cells displayed high expression levels, PC-3 cells disclosed significantly lower levels. These findings provided a unique opportunity to evaluate the biological role of miR-375 in PCa cells, using two opposing, yet complementary strategies. Intriguingly, both anti-miR-375 transfection in 22Rv1 cells (causing 68\% reduction in miR-375 expression levels) and forced miR-375 expression in PC-3 cells attenuated the malignant phenotype, whereas in RPWE-1 cells, forced miR-375 expression did not cause significant phenotypic alterations. Thus, while in $22 \mathrm{Rv} 1$ cells an oncogenic 


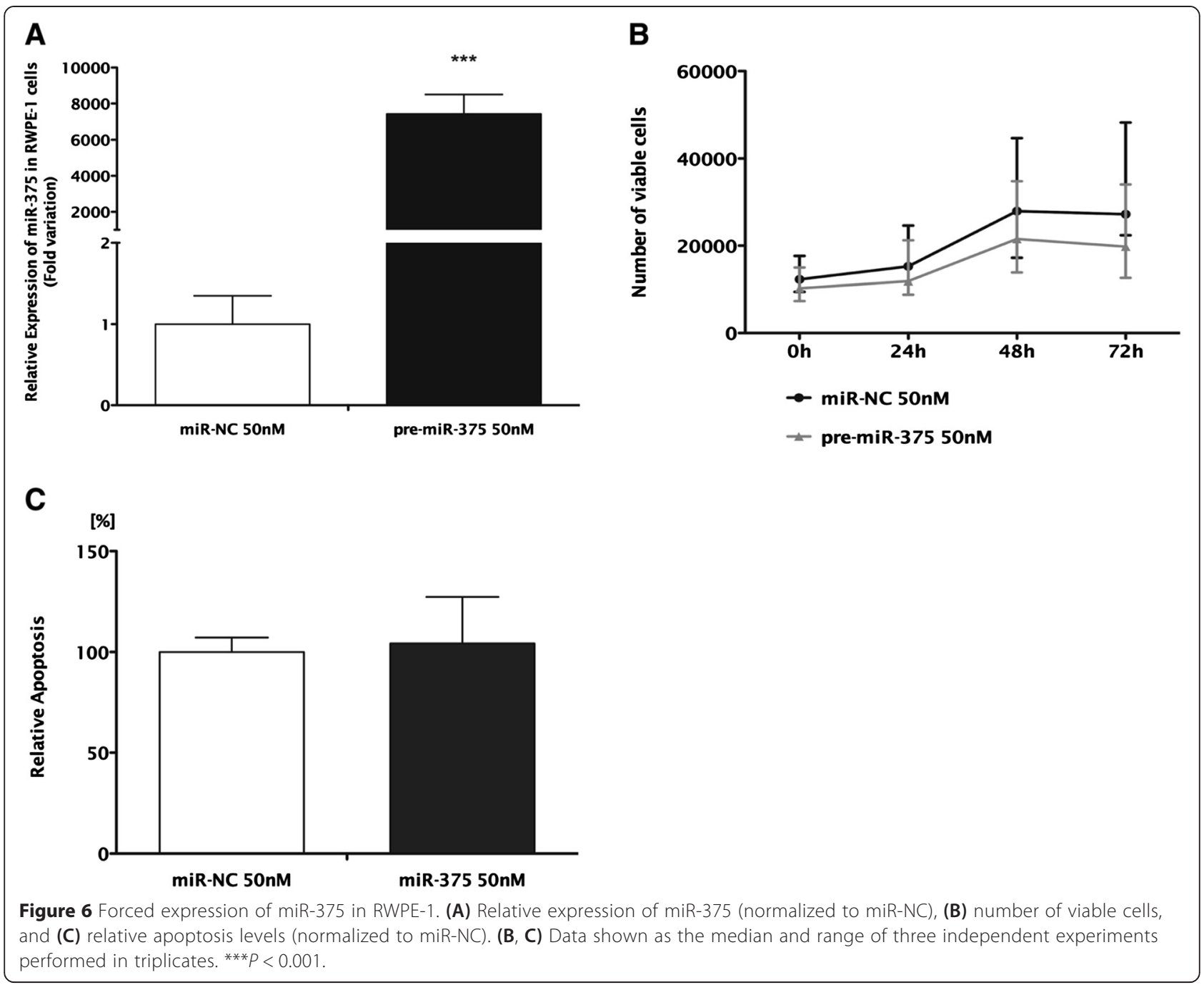

role for miR-375 is suggested, a tumor-suppressive function is implied for PC-3 cells. Considering that miRNAs might play an oncogenic or tumor-suppressive role depending on the cellular context in different tumors [19,22], this observation would not be surprising, except for the fact that it occurred in the same tumor model. However, due to the widely acknowledged heterogeneity of $\mathrm{PCa}$, it may be reasonable to assume that in different prostate cancers (herein represented by different cell lines) miR-375 could play antagonistic roles.

Considering the potential dual role of miR-375 in PCa, the expression of 61 cancer-related genes, involved in critical cellular pathways, was assessed in PC-3 and 22Rv1 transfected cells. The panel of altered genes was, indeed, different in each cell line, as it would be expected from the results of the phenotypic assays and the baseline expression levels of miR-375. These results might also be explained by the acknowledged 'promiscuity' of miRNAs, as a single miRNA may target several different gene transcripts in a time- and model-dependent manner [10,30]. In the expression analysis, retinoblastoma $1(R B 1)$ and cyclin D2 (CCND2) surfaced as the most deregulated genes in each cell line. Remarkably, CCND2 expression was found to be decreased in primary PCa samples, consistent with a putative target of miR-375 (which is overexpressed in those samples), whereas $R B 1$ was overexpressed in the same set of primary tumors. The former result is in line with previous reports on CCND2 downregulation in $\mathrm{PCa}[31,32]$ whereas the latter contradicts previous observations concerning RB1 downregulation in $\mathrm{PCa}$ [33]. However, it should be emphasized that in addition to miR-375, other epigenetic and/or genetic mechanisms, eventually more relevant in vivo, might be involved in RB1 expression regulation in PCa. Furthermore, the luciferase assay confirmed that CCND2 is a target of miR-375 as CCND2 transcript levels were significantly reduced after miR-375 forced expression. Interestingly, CCND2 is a key element in cell cycle regulation, and this pathway was found as the most relevant in which miR-375 was implicated, in gene ontology enrichment analysis of both transfected cell lines. It should be 

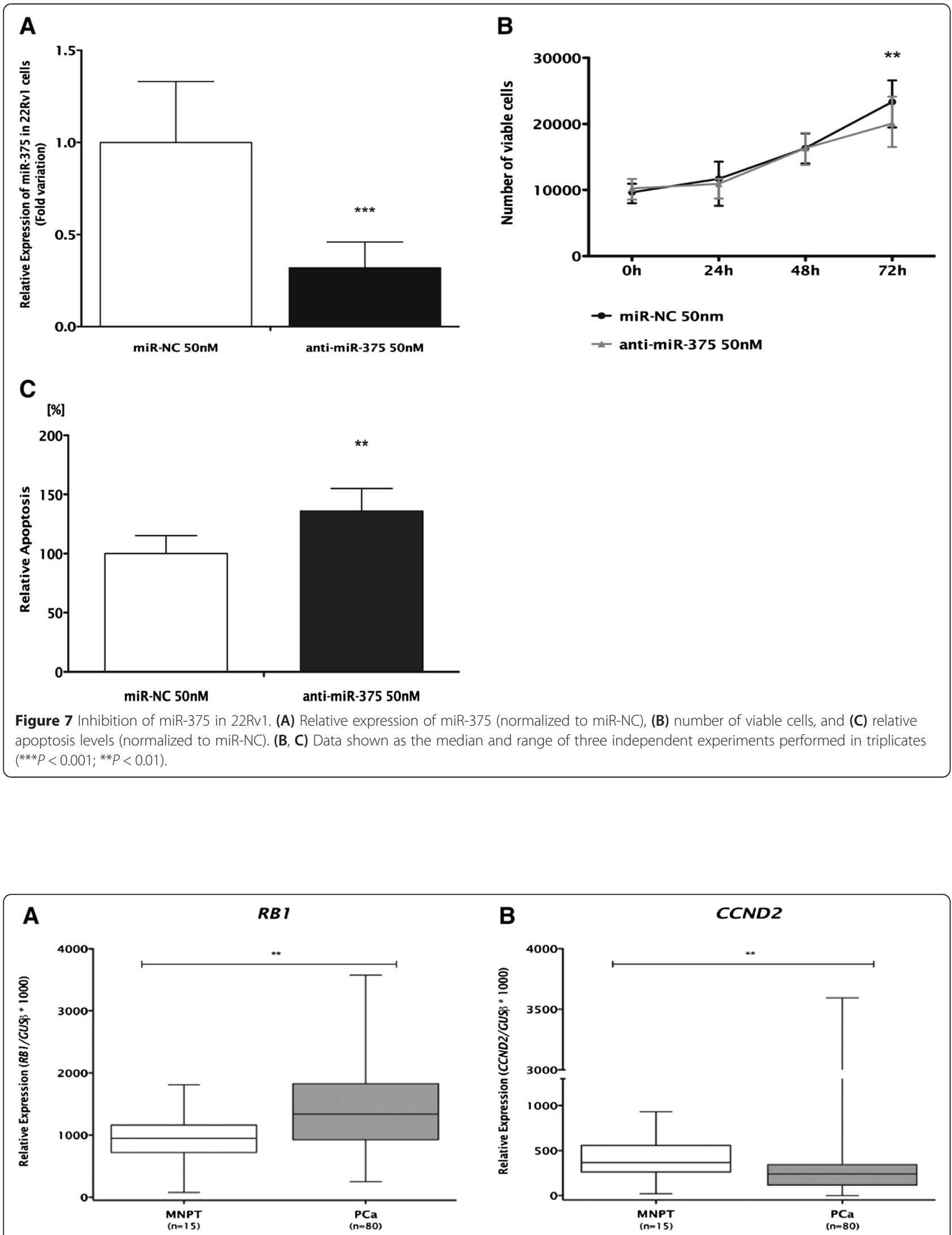

Figure 8 Expression levels of potential miR-375 targets for array validation (A) $R B 1$ and (B) CCND2 $(* * P<0.01)$. 


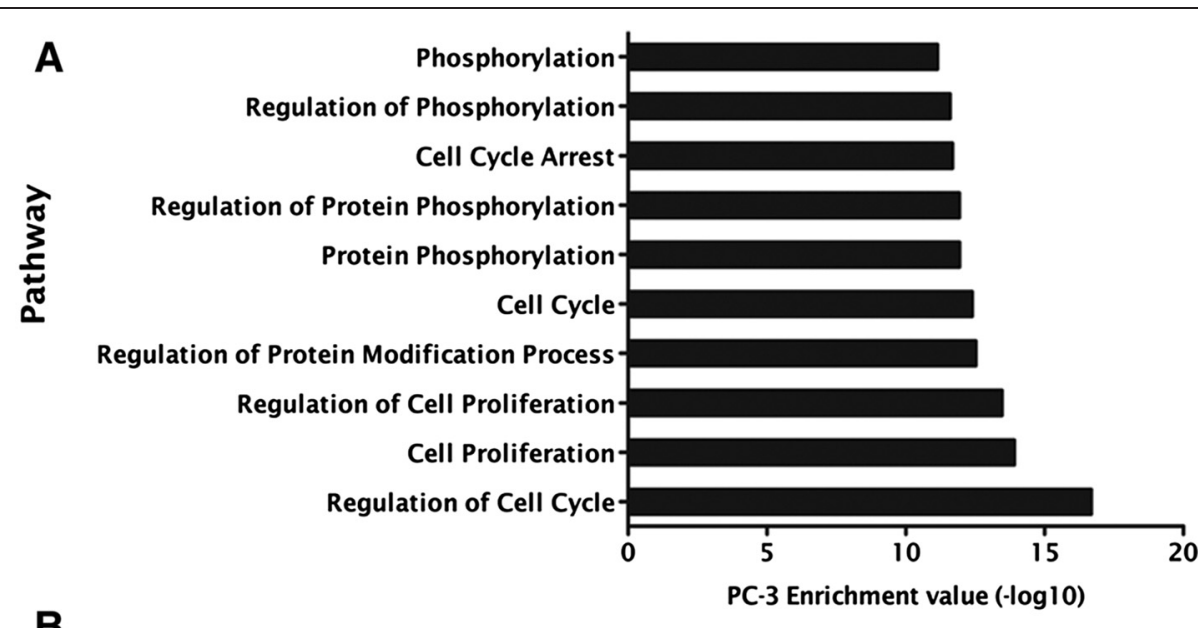

B

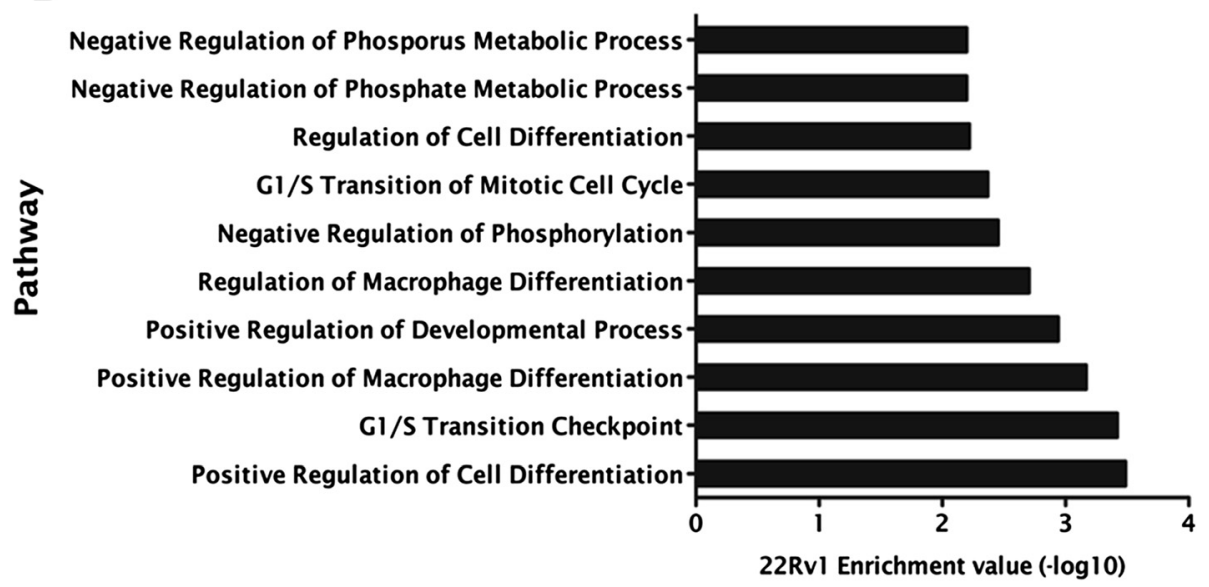

Figure 9 GO enrichment analysis: pathways (A) downregulated in PC-3 and (B) upregulated in 22Rv1 transfected cells.

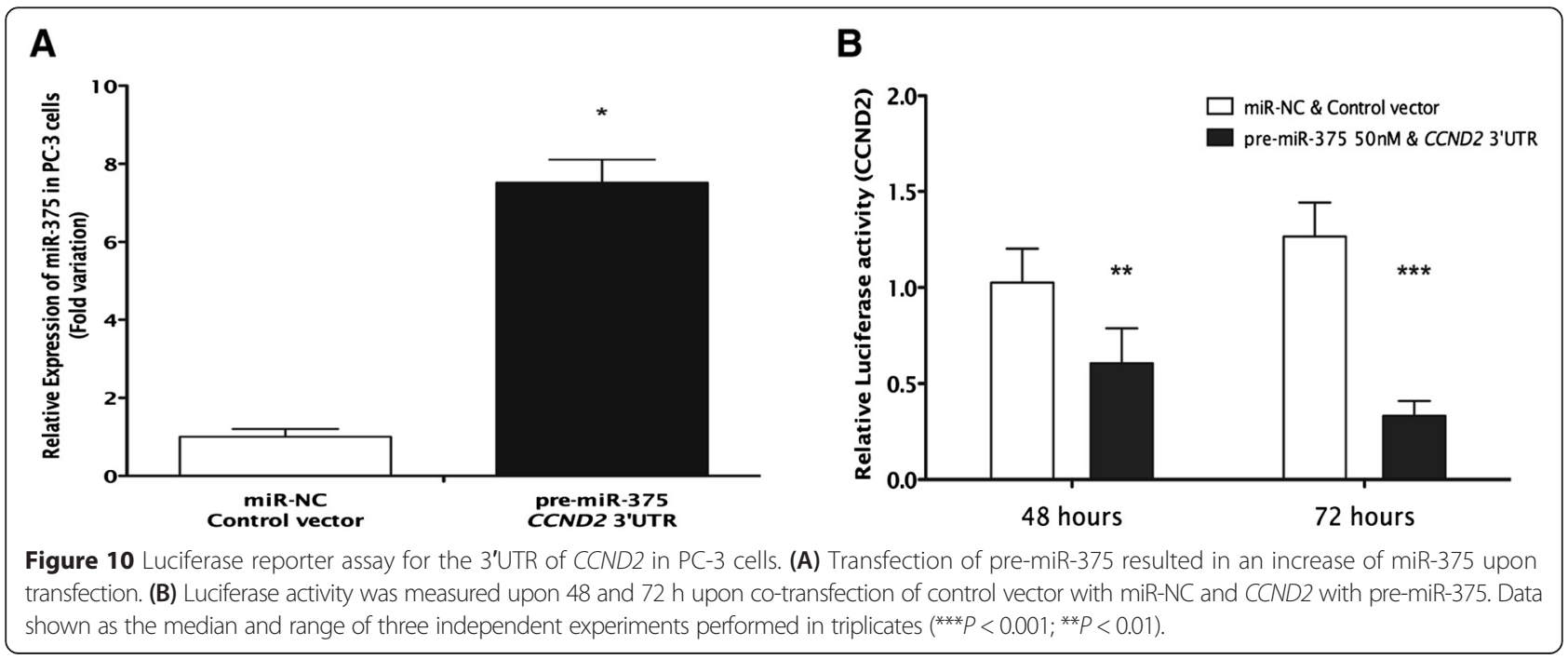


recalled, however, that downregulation of $C C N D 2$ has also been associated with aberrant promoter methylation [31] and, thus, different epigenetic mechanisms may act in concert to accomplish CCND2 silencing in PCa.

Our results thus suggest that miR-375 plays a dual role in $\mathrm{PCa}$, acting either as an oncomiR or a tumorsuppressor miRNA, depending on the cellular context. It is noteworthy that $\mathrm{PCa}$ cell lines with the highest miR-375 expression levels are androgen-responsive (22Rv1 and LNCaP), whereas androgen-independent (DU145 and PC-3) cells display the lowest levels. Strikingly, these results are in line with previous reports that associate DNMT activity, promoter methylation of miR-375 and androgens [34]. Interestingly, although derived from metastasis, 22Rv1 and LNCaP cell lines display the miR-375 expression profile typical of primary PCa from both series analyzed, and these are also androgen responsive as they represent clinically localized, androgen-ablation therapy-naïve tumors. Thus, the cellular context in which miR-375 may act as oncomiR or tumor suppressor is likely to be conditioned by androgen receptor regulation. Examples of miRNAs that may act as tumor suppressors or oncomiRs, depending on the tumor model, are acknowledged (for example, cluster miR-191/ 425 in breast cancer depending on estrogen status) [35]. However, this seems to be a previously unrecognized event in which a miRNA may have that dual role in the same tumor model, depending on the stage of tumor progression and hormonal environment. It is tempting to speculate whether in the same tumor, along its progression, miR-375 may act initially as an oncomiR and later as a tumor suppressor, targeting different genes as disease progresses. The mechanisms underlying this putative alteration remain elusive but it could derive from the significant alteration of the gene expression landscape of $\mathrm{PCa}$ cells along disease progression [36].

\section{Conclusions}

In conclusion, our data provides further insight into miRNAs overexpression in $\mathrm{PCa}$, suggesting that miR-375 upregulation might be act as oncomiR at the initial steps of prostate carcinogenesis, a role that could be impaired as PCa progresses, probably due to the cumulative genetic and epigenetic alterations endured by cancer cells. We provide evidence that miR-375 deregulation disturbs several critical cellular pathways, especially cell cycle regulation, eventually through CCND2 targeting, which may, at the least partially, explain the frequent downregulation of CCND2 in primary $\mathrm{PCa}$.

\section{Methods}

\section{Patients and sample collection}

Primary tumors from 119 patients harboring clinically localized prostate adenocarcinoma were prospectively collected after diagnosis and primary treatment with radical prostatectomy at Portuguese Oncology Institute, Porto, Portugal. A set of 15 MNPTs was collected from prostatic peripheral zone of bladder cancer patients submitted to cystoprostatectomy. All tissue specimens were promptly frozen immediately after surgery. Upon histological confirmation of tumor or normal prostate tissue, fresh-frozen tissue fragments were trimmed to enhance yield of target cells $(>70 \%)$. Histological slides from formalin-fixed paraffin-embedded tissue fragments were also routinely obtained from the surgical specimens and assessed for Gleason score and TNM stage. Relevant clinical data was collected from clinical charts. Informed consent was obtained from all participants, according to institutional regulations. This study was approved by the institutional review board [Comissão de Ética para a Saúde-(CES-IPOPFG-EPE 205/2013)] of Portuguese Oncology Institute - Porto, Portugal.

\section{Cell culture}

Human PCa cell lines available in our lab (LNCaP, 22Rv1, DU145 and PC-3) and a non-malignant prostate cell line (RWPE-1, kindly provided by Prof. Margarida Fardilha, University of Aveiro, Portugal) were used in this study. All cell lines were cultured using recommended medium supplemented with $10 \%$ of fetal bovine serum and $1 \%$ of penicillin-streptomycin (FBS; GIBCO, Invitrogen, Carlsbad, CA, USA) and maintained at $37^{\circ} \mathrm{C}$ and $5 \% \mathrm{CO}_{2}$ in a humidified chamber. All cell lines were routinely tested for contamination by Mycoplasma spp. using a specific multiplex PCR (PCR Mycoplasma Detection Set, Clontech Laboratories Inc., Mountain View, CA, USA).

\section{Total RNA extraction}

Total RNA from clinical samples and cell lines was obtained by suspension in TRIzol $^{\circ}$ reagent (Invitrogen, Carlsbad, CA, USA) and, after adding chloroform, total RNA was purified from the aqueous phase of TRIzol ${ }^{\circ}$ extract using PureLink ${ }^{\mathrm{Th}}$ RNA Mini Kit (Invitrogen, Carlsbad, CA, USA) following the manufacturer's recommendations. RNA concentration, purity, and integrity of samples were determined on a Nanodrop ND-1000 spectrophotometer (NanoDrop Technologies, Wilmington, DE, USA) and electrophoresis.

\section{MicroRNAs global expression}

Global miRNAs expression was assessed in ten $\mathrm{PCa}$ and four MNPT using microRNA Ready-to-Use PCR Human Panel (I + II) v2.0 (Exiqon, Vedbaek, Denmark), consisting of 739 miRNAs in total. RNA samples were submitted to cDNA synthesis using miRCURY LNA $^{\mathrm{m}}$ Universal RT microRNA PCR (Exiqon, Vedbaek, Denmark) following manufacturer's instructions. Briefly, for each sample, $4 \mu \mathrm{L}$ of $5 \times$ reaction buffer, $9-\mu \mathrm{L}$ nuclease-free water, $2 \mu \mathrm{L}$ of 
enzyme mix, $1 \mu \mathrm{L}$ of synthetic spike in, and $4 \mu \mathrm{L}$ of previously concentration-adjusted RNA. Tubes were then vortexed gently, and reverse transcription was performed in Veriti ${ }^{\circledR}$ Thermal Cycler (Applied Biosystems, Foster City, CA, USA). Protocol consisted of incubation for $60 \mathrm{~min}$ at $42^{\circ} \mathrm{C}$, followed by $5 \mathrm{~min}$ at $95^{\circ} \mathrm{C}$. Global expression was performed in a LightCycler 480 Instrument (Roche Diagnostics, Manheim, Germany) according to the manufacturer's conditions. Data was analyzed using GenEX software (MultiD Analyses AB, Göteburg, Sweden). Then, data were analyzed using the comparative $\mathrm{Ct}$ method [37]. Median value of reference genes was used for normalization, and miRNAs with fold change higher than 1.5 were classified as overexpressed in $\mathrm{PCa}$ compared to MNPT.

\section{Validation of microRNAs expression}

cDNA was synthesized from $119 \mathrm{PCa}, 15 \mathrm{MNPT}$ and 5 prostate cell lines, using miRCURY LNA ${ }^{\mathrm{sm}}$ Universal RT microRNA PCR (Exiqon, Vedbaek, Denmark), following the manufacturer's instructions, as described above. Samples were then eluted $80 \times$ in nuclease-free water. MiRNAs' levels were evaluated using specific primers (microRNA LNA $^{\mathrm{TM}}$ PCR primer set, Exiqon, Vedbaek, Denmark) according to the manufacturer's recommendations. In each well, $4 \mu \mathrm{L}$ of diluted cDNA were mixed with $1 \mu \mathrm{L}$ of specific miRNAs qPCR primers (Exiqon, Vedbaek, Denmark), $2 \mu \mathrm{L}$ of ROX reference dye (Invitrogen, Carlsbad, CA, USA) and $5 \mu \mathrm{L}$ of $\mathrm{SYBR}^{\ominus}$ Green Master mix (Exiqon, Vedbaek, Denmark). Protocol consisted in a denaturation step at $95^{\circ} \mathrm{C}$ for $10 \mathrm{~min}$, followed by 40 amplification cycles at $95^{\circ} \mathrm{C}$ for $10 \mathrm{~s}$ and $60^{\circ} \mathrm{C}$ for $1 \mathrm{~min}$. As previously mentioned, melting curve analysis was also performed at the end of the procedure according to instrument's manufacturer recommendations. Each 96-well plate included multiple non-template controls and serial dilutions $(10 \times)$ of cDNA obtained from human prostate RNA (Ambion, Invitrogen, Carlsbad, CA, USA) was used to construct a standard curve for each plate. All experiments were run in triplicates in a 7500 Sequence Detection System (Applied Biosystems, Foster City, CA, USA). Considering the results from global analysis, it was decided to use the reference gene with less variation (miR-423-5p) among samples for normalization of validation data. Relative expression of miRNAs was determined as target gene mean quantity/reference gene mean quantity. Values were then multiplied by 1,000 for easier tabulation.

\section{MicroRNAs transient transfection}

miR-375 was transiently transfected in PC-3 and RWPE-1 cells with a Pre-miR ${ }^{\mathrm{m}}$ miRNA precursor (pre-miR-375, PM10327, Applied Biosystems, Foster City, CA, USA) and an Anti-miR ${ }^{\mathrm{TM}}$ miRNA inhibitor (anti-miR-375, AM10327, Applied Biosystems, Foster City, CA, USA) was transfected for $22 \mathrm{Rv} 1$ cells. A miRNA negative control was used as control in all experiments (miR-NC, AM17010, Applied Biosystems, Foster City, CA, USA). Cells were seeded under standard conditions in six-well and 96-well plates for $24 \mathrm{~h}$ before transfection, reaching $30 \%$ to $50 \%$ confluence. In these experiments, pre-miR-375, anti-miR-375, and miR-NC concentration was $50 \mathrm{nM}$. Oligofectamine ${ }^{\mathrm{TM}}$ reagent (Invitrogen, Carlsbad, CA, USA) was used under conditions indicated by the manufacturer. Cells were then incubated at $37^{\circ} \mathrm{C}$ and $5 \% \mathrm{CO}_{2}$ in a humidified chamber for $72 \mathrm{~h}$ upon transfection. At $72 \mathrm{~h}$, forced expression or silencing of miR-375 were confirmed by RT-qPCR.

\section{Cell viability assay}

To evaluate the impact of in vitro transfection of miR-375 in PCaer cell lines, 3-(4,5-dimethylthiazol-2-yl)-2,5diphenyltetrazolium (MTT; Sigma-Aldrich, Schnelldorf, Germany) assay was performed in 96-well plates. Briefly, cells were incubated with $10 \% \mathrm{MTT}$ at $5 \mathrm{mg} / \mathrm{mL}$ in a humidified chamber for 24,48 , and $72 \mathrm{~h}$ after transfection. Reaction was stopped by removal of MTT and addition of $100 \mu \mathrm{L}$ DMSO (Sigma-Aldrich, Schnelldorf, Germany) per well. Finally, plates were shaken for 15 min for complete dissolution. Absorbance levels were measured using a microplate reader (Fluostar Omega, BMG Labtech, Offenburg, Germany) at $540 \mathrm{~nm}$ with background deduction at $630 \mathrm{~nm}$. Number of viable cells was obtained using the following formula: (OD experiment $\times$ Mean number of cells at $0 \mathrm{~h}$ )/Mean OD at $0 \mathrm{~h}$. Three biologically independent experiments were performed, comprising methodological triplicates for each experiment.

\section{Apoptosis assay}

Apoptosis was assessed using the APOPercentage ${ }^{\text {тM }}$ kit (Biocolor Ltd., Belfast, Northern Ireland, UK). Cell lines were seeded under the same conditions as described for MTT assay and, after $72 \mathrm{~h}$ incubation, apoptosis assay was performed according to the manufacturer's instructions. Quantification of apoptosis was achieved by measuring the optical density of the released dye at $550 \mathrm{~nm}$ with background deduction at $620 \mathrm{~nm}$ using a FLUOstar Omega microplate reader. To normalize the OD obtained for the apoptosis assays relatively to cell number, OD of cell viability assay at $72 \mathrm{~h}$ was used. Results were expressed as ratio of transfected cells OD to miR-NC OD (set as 100\%).

\section{Invasion assay}

Invasion ability of PC-3 transfected cells was analyzed using BD Biocoat ${ }^{\mathrm{TM}}$ Matrigel Invasion Chambers (BD Biosciences, Franklin Lakes, NJ, USA) according to the manufacturer's protocol. Briefly, cells were seeded and then transfected in six-well plates. After $48 \mathrm{~h}$ of transfection, cells were 
trypsinized and seeded in serum-free culture medium in Matrigel inserts and allowed to invade for $24 \mathrm{~h}$ at $37^{\circ} \mathrm{C}$ and $5 \% \mathrm{CO}_{2}$ in a humidified chamber. Medium with serum was used as chemoattractant. Then, non-invasive cells were removed from the top of the membranes and cells that invaded were fixed with methanol and stained with DAPI. Invasive cells were manually counted in a fluorescence microscope, and results were displayed as a percentage of cells that crossed the membrane (invading cells) relative to miR-NC.

\section{Identification of potential miR-375 target genes}

To determine whether miR-375 was implicated in regulation of selected genes involved in cell cycle, apoptosis, DNA repair, mTOR, or MAPK/ERK pathways, a custom array panel (Roche Applied Science, Manheim, Germany) was designed for quantification of selected gene expression. Total RNA was extracted from all cell lines using TRIzol $^{\circ}$ (Invitrogen, Carlsbad, CA, USA) according to the manufacturer's instructions and cDNA synthesis was performed using Transcriptor High Fidelity cDNA Synthesis Kit (Roche, Manheim, Germany) according to the manufacturer's instructions. Expression levels were determined by real-time PCR in a LightCycler 480 (Roche Diagnostics, Manheim, Germany) and the amount of mRNA was normalized using GUSB, TFRC, and $18 S$ as endogenous controls. The comparative Ct method [37] was used to calculate fold-difference in gene expression between mir-375 transfected cells and respective miR-NC.

\section{Gene ontology enrichment analysis}

Gene ontology enrichment (GOE) analysis was performed to ascertain which biological processes are regulated by miR-375 in PCa cell lines. AmiGO database [38] was used, and statistical analysis was performed using $\mathrm{R}$ program based on hypergeometric distribution followed by Fisher's exact test [39].

\section{Expression of potential target genes in clinical samples}

Following gene selection, mRNA levels were confirmed in the same group of tissue samples previously indicated. A total of $300 \mathrm{ng}$ was reverse transcribed and amplified using TransPlex ${ }^{\circ}$ Whole Transcriptome Amplification Kit (Sigma-Aldrich ${ }^{\circ}$, Schnelldorf, Germany) with subsequent purification using QIAquick $^{\circ}$ PCR Purification Kit (QIAGEN, Hilden, Germany), according to the manufacturer's instructions. Expression levels were evaluated using TaqMan ${ }^{\circ}$ Gene Expression Assays (Applied Biosystems, Foster City, CA, USA), and GUSB was used as a reference gene for normalization, according to the formula: Relative expression $=($ Target gene mean quantity $/$ Reference gene mean quantity). Ratios were then multiplied by 1,000 for easier tabulation. Each plate included multiple non-template controls and serial dilutions $(10 \times)$ of a cDNA obtained from human prostate RNA (Ambion) were used to construct a standard curve for each plate. All experiments were run in triplicates.

\section{Luciferase assay}

A reporter construct containing a binding site at CCND2 3'UTR for miR-375 (GeneCopoeia, Rockville, MD, USA) was introduced into PC-3 cells using Turbofectin 8.0 transfection reagent (Origene, Rockville, MD, USA). A vector without CCND2 3'UTR (GeneCopoeia) was used as experiment control. Vectors were co-transfected along with pre-miR-375 as described. Luciferase activity was assessed with the Secrete-Pair ${ }^{\text {Tix }}$ Dual Luminescence Assay Kit (GeneCopoeia, Rockville, MD, USA) according to the manufacturer's instructions. Experiments were performed in triplicates at 48 and $72 \mathrm{~h}$ following cotransfection. At $72 \mathrm{~h}, \mathrm{miR}-375$ levels were measured by RT-qPCR to confirm its forced or silenced expression.

\section{TCGA data meta-analysis in prostate cancer patients}

TCGA was used to obtain data on miRNA expression and clinical information, when available, from $\mathrm{PCa}$ and matched normal tissue samples [40]. All miRNA expression data from samples hybridized by the University of North Carolina, Lineberger Comprehensive Cancer Center, using Illumina HiSeq 2000 miRNA Sequencing, were downloaded from TCGA data matrix (http://tcgadata.nci.nih.gov/tcga/tcgaDownload.jsp). This dataset included $326 \mathrm{PCa}$ and 50 matched normal patient samples. To prevent duplicates, when there was more than one portion per patient, median values were used. The provided value was pre-processed and normalized according to 'level 3' specifications of TCGA (see http://cancergenome.nih.gov/ for details). Clinical data of each patient was provided by the Biospecimen Core Resources (BCRs). This data is available for download through TCGA data matrix (http://tcga-data.nci.nih.gov/tcga/data AccessMatrix.htm).

\section{Statistical analysis}

The Shapiro-Wilk's $W$ test allowed for the examination of the appropriateness of a normal distribution assumption for each of the parameters (data not shown). Comparisons between two groups were then performed using nonparametric Mann-Whitney $U$-test. $P$ values were considered statistically significant if lower than 0.05 .

Correlation between miRNAs' expression was measured by the Spearman's correlation coefficient $(r)$. Differences in miR-375 expression between N0 and N1 lymph node stage groups were assessed by Student's $t$-test.

Statistical analysis was performed SPSS 20.0 for Mac (IBM-SPSS Inc., Chicago, IL, USA), and graphs were built using GraphPad Prism 5.0 software for Mac (GraphPad Software Inc., La Jolla, CA, USA). 


\section{Additional files}

Additional file 1: Table S1. Clinical and pathological data of patients included in this study for miR-32 and miR-182.

Additional file 2: Figure S1. Validation of expression levels of (A) miR-32, (B) miR-182 (***P $<0.001$; ns, non-significant).

Additional file 3: Figure S4. Expression of miR-32 and miR-182 is increased in prostate cancer in patients from TCGA.

Additional file 4: Figure S2. Invasion assay in PC-3 cell line. Representative display of (A) miR-NC cells and (B) pre-miR-375 $50 \mathrm{nM}$ transfected cells.

Additional file 5: Figure S3. Potential miR-375 target cancer-related genes in 22Rv1 and PC-3 transfected cells, normalized to miR-NC.

\section{Competing interests}

The authors declare that they have no competing interests.

\section{Authors' contributions}

$P C P, R H$, and $\mathrm{CJ}$ contributed to experimental design. PCP and FQV designed and executed in vitro assays and analysis. PCP and JTF performed RTq-PCR analysis. JRC performed global expression analysis of miRNAs. PCP and JRC performed luciferase assays. JO collected relevant clinical information. BMC and CSG performed TCGA research and analysis. RH performed tumor collection and histopathological classification. PCP, RH, and CJ wrote the manuscript with input from co-authors. All authors read and approved the final manuscript.

\section{Authors' information}

Rui Henrique and Carmen Jerónimo are joint senior authors.

\section{Acknowledgments}

The authors would like to acknowledge funding attributed to this study, namely research grants from Research Center of Portuguese Oncology Institute - Porto (CI-IPOP 4-2012) and by the Federal funds through Programa Operacional Temático Factores de Competitividade (COMPETE) with co-participation from the European Community Fund (FEDER) and by the National funds through Fundação para a Ciência e Tecnología (FCT) under the projects EXPL/BIM-ONC/0556/2012. FQV and JRC were or are supported by FCT-Fundação para a Ciência e a Tecnologia grants (SFRH/ BD/70564/2010 and SFRH/BD/71293/2010, respectively).

\section{Author details}

${ }^{1}$ Cancer Biology and Epigenetics Group - Research Center (Lab3), Portuguese Oncology Institute - Porto (IPO-Porto), Rua Dr. António Bernardino de Almeida, Porto 4200-072, Portugal. ${ }^{2}$ School of Allied Health Sciences (ESTSP), Polytechnic of Porto, Rua Valente Perfeito 322, Vila Nova de Gaia 4400-330, Portugal. ${ }^{3}$ Department of Urology, Portuguese Oncology Institute, Rua Dr. António Bernardino de Almeida, Porto 4200-072, Portugal. ${ }^{4}$ Department of Pathology, Portuguese Oncology Institute - Porto, Rua Dr. António Bernardino de Almeida, Porto 4200-072, Portugal. ${ }^{5}$ Life and Health Sciences Research Institute (ICVS), School of Health Sciences, University of Minho, Rua da Universidade, 4704-553 Braga, Portugal. 'ICVS/3B's - PT Government Associate Laboratory, Universidade do Minho, Campus de Gualtar, 4710-057 Guimarães, Braga, Portugal. ${ }^{7}$ Department of Pathology and Molecular Immunology, Institute of Biomedical Sciences Abel Salazar (ICBAS), University of Porto, Rua de Jorge Viterbo Ferreira 228, Porto 4050-313, Portugal.

\section{Received: 1 December 2014 Accepted: 19 March 2015}

\section{Published online: 10 April 2015}

\section{References}

1. Ferlay J, Steliarova-Foucher E, Lortet-Tieulent J, Rosso S, Coebergh JW, Comber $\mathrm{H}$, et al. Cancer incidence and mortality patterns in Europe: estimates for 40 countries in 2012. Eur J Cancer. 2013;49(6):1374-403. doi:10.1016/j.ejca.2012.12.027.

2. Siegel R, Ma J, Zou Z, Jemal A. Cancer statistics, 2014. CA Cancer J Clin. 2014;64(1):9-29. doi:10.3322/caac.21208.

3. Hricak H, Scardino PT. Prostate cancer. Contemporary issues in cancer imaging. Cambridge, UK. New York: Cambridge University Press; 2009.
4. Jeronimo C, Bastian PJ, Bjartell A, Carbone GM, Catto JW, Clark SJ, et al. Epigenetics in prostate cancer: biologic and clinical relevance. Eur Urol. 2011;60(4):753-66. doi:10.1016/j.eururo.2011.06.035

5. Porkka KP, Pfeiffer MJ, Waltering KK, Vessella RL, Tammela TL, Visakorpi T. MicroRNA expression profiling in prostate cancer. Cancer Res. 2007:67(13):6130-5. doi:10.1158/0008-5472. CAN-07-0533.

6. Croce CM. Causes and consequences of microRNA dysregulation in cancer Nat Rev Genet. 2009;10(10):704-14. doi:10.1038/nrg2634.

7. Iorio MV, Croce CM. MicroRNAs in cancer: small molecules with a huge impact. J Clin Oncol. 2009:27(34):5848-56. doi:10.1200/JCO.2009.24.0317.

8. Kozomara A, Griffiths-Jones S. miRBase: annotating high confidence microRNAs using deep sequencing data. Nucleic Acids Res. 2014;42(Database issue):D68-73. doi:10.1093/nar/gkt1181

9. van Rooij E, Olson EN. MicroRNA therapeutics for cardiovascular disease: opportunities and obstacles. Nat Rev Drug Discov. 2012;11(11):860-72. doi:10.1038/nrd3864

10. Iorio MV, Croce CM. MicroRNA dysregulation in cancer: diagnostics, monitoring and therapeutics. A comprehensive review. EMBO Mol Med. 2012;4(3):143-59. doi:10.1002/emmm.201100209.

11. Davalos V, Esteller M. MicroRNAs and cancer epigenetics: a macrorevolution. Curr Opin Oncol. 2010;22(1):35-45. doi:10.1097/CCO.0b013e328333dcbb.

12. Catto JW, Alcaraz A, Bjartell AS, De Vere WR, Evans CP, Fussel S, et al. MicroRNA in prostate, bladder, and kidney cancer: a systematic review. Eur Urol. 2011;59(5):671-81. doi:10.1016/j.eururo.2011.01.044.

13. Coppola V, De Maria R, Bonci D. MicroRNAs and prostate cancer. Endocr Relat Cancer. 2010;17(1):F1-F17. doi:10.1677/ERC-09-0172.

14. Shen MM, Abate-Shen C. Molecular genetics of prostate cancer: new prospects for old challenges. Genes Dev. 2010;24(18):1967-2000. doi:10.1101/gad.1965810.

15. Lewis $H$, Lance $R$, Troyer $D$, Beydoun $H$, Hadley M, Orians J, et al. miR-888 is an expressed prostatic secretions-derived microRNA that promotes prostate cell growth and migration. Cell Cycle. 2014;13(2):227-39. doi:10.4161/cc.26984

16. Sun R, Fu X, Li Y, Xie Y, Mao Y. Global gene expression analysis reveals reduced abundance of putative microRNA targets in human prostate tumours. BMC Genomics. 2009:10:93. doi:10.1186/1471-2164-10-93.

17. Lockhart DJ, Winzeler EA. Genomics, gene expression and DNA arrays. Nature. 2000;405(6788):827-36. doi:10.1038/35015701.

18. Tsuchiyama K, Ito H, Taga M, Naganuma S, Oshinoya $Y$, Nagano K, et al. Expression of microRNAs associated with Gleason grading system in prostate cancer: miR-182-5p is a useful marker for high grade prostate cancer. Prostate. 2013;73(8):827-34. doi:10.1002/pros.22626.

19. Cheng HH, Mitchell PS, Kroh EM, Dowell AE, Chery L, Siddiqui J, et al. Circulating microRNA profiling identifies a subset of metastatic prostate cancer patients with evidence of cancer-associated hypoxia. PLoS One. 2013;8(7), e69239. doi:10.1371/journal.pone.0069239.

20. Nguyen $\mathrm{HC}$, Xie W, Yang M, Hsieh CL, Drouin S, Lee GS, et al. Expression differences of circulating microRNAs in metastatic castration resistant prostate cancer and low-risk, localized prostate cancer. Prostate. 2013;73(4):346-54. doi:10.1002/pros.22572.

21. Huang X, Yuan T, Liang M, Du M, Xia S, Dittmar R, et al. Exosomal miR-1290 and miR-375 as prognostic markers in castration-resistant prostate cancer. Eur Urol. 2015:67(1):33-41. doi:10.1016/j.eururo.2014.07.035.

22. Szafranska AE, Davison TS, John J, Cannon T, Sipos B, Maghnouj A, et al. MicroRNA expression alterations are linked to tumorigenesis and non-neoplastic processes in pancreatic ductal adenocarcinoma. Oncogene. 2007;26(30):4442-52. doi:10.1038/sj.onc.1210228

23. Ladeiro Y, Couchy G, Balabaud C, Bioulac-Sage P, Pelletier L, Rebouissou S, et al. MicroRNA profiling in hepatocellular tumors is associated with clinical features and oncogene/tumor suppressor gene mutations. Hepatology. 2008;47(6):1955-63. doi:10.1002/hep.22256

24. Avissar M, Christensen BC, Kelsey KT, Marsit CJ. MicroRNA expression ratio is predictive of head and neck squamous cell carcinoma. Clin Cancer Res. 2009;15(8):2850-5. doi:10.1158/1078-0432. CCR-08-3131.

25. Tsukamoto Y, Nakada C, Noguchi T, Tanigawa M, Nguyen LT, Uchida T, et al. MicroRNA-375 is downregulated in gastric carcinomas and regulates cell survival by targeting PDK1 and 14-3-3zeta. Cancer Res. 2010;70(6):2339-49. doi:10.1158/0008-5472. CAN-09-2777.

26. Ding L, Xu Y, Zhang W, Deng Y, Si M, Du Y, et al. MiR-375 frequently downregulated in gastric cancer inhibits cell proliferation by targeting JAK2. Cell Res. 2010;20(7):784-93. doi:10.1038/cr.2010.79. 
27. Szczyrba J, Nolte E, Wach S, Kremmer E, Stohr R, Hartmann A, et al. Downregulation of Sec23A protein by miRNA-375 in prostate carcinoma. Mol Cancer Res. 2011;9(6):791-800. doi:10.1158/1541-7786. MCR-10-0573.

28. Selth LA, Townley S, Gillis JL, Ochnik AM, Murti K, Macfarlane RJ, et al. Discovery of circulating microRNAs associated with human prostate cancer using a mouse model of disease. Int J Cancer. 2012;131(3):652-61. doi:10.1002/ijc.26405

29. Wach S, Nolte E, Szczyrba J, Stohr R, Hartmann A, Orntoft T, et al. MicroRNA profiles of prostate carcinoma detected by multiplatform microRNA screening. Int J Cancer. 2012;130(3):611-21. doi:10.1002/ijc.26064.

30. Nam JW, Rissland OS, Koppstein D, Abreu-Goodger C, Jan CH, Agarwal V, et al. Global analyses of the effect of different cellular contexts on microRNA targeting. Mol Cell. 2014;53(6):1031-43. doi:10.1016/.j.molcel.2014.02.013.

31. Costa VL, Henrique R, Jeronimo C. Epigenetic markers for molecular detection of prostate cancer. Dis Markers. 2007:23(1-2):31-41.

32. Kobayashi T, Nakamura E, Shimizu Y, Terada N, Maeno A, Kobori G, et al. Restoration of cyclin D2 has an inhibitory potential on the proliferation of LNCaP cells. Biochem Biophys Res Commun. 2009;387(1):196-201. doi:10.1016/j.bbrc.2009.06.146.

33. Bettendorf O, Schmidt H, Staebler A, Grobholz R, Heinecke A, Boecker W, et al. Chromosomal imbalances, loss of heterozygosity, and immunohistochemical expression of TP53, RB1, and PTEN in intraductal cancer, intraepithelial neoplasia, and invasive adenocarcinoma of the prostate. Genes, Chromosomes Cancer. 2008:47(7):565-72. doi:10.1002/gcc.20560.

34. Chu M, Chang Y, Li P, Guo Y, Zhang K, Gao W. Androgen receptor is negatively correlated with the methylation-mediated transcriptional repression of miR-375 in human prostate cancer cells. Oncol Rep. 2014:31(1):34-40. doi:10.3892/or.2013.2810

35. Di Leva G, Piovan C, Gasparini P, Ngankeu A, Taccioli C, Briskin D, et al. Estrogen mediated-activation of miR-191/425 cluster modulates tumorigenicity of breast cancer cells depending on estrogen receptor status. PLoS Genet. 2013;9(3), e1003311. doi:10.1371/journal.pgen.1003311.

36. DeVita VT, Lawrence TS, Rosenberg SA. Cancer: principles and practice of oncology. 5th ed. Philadelphia: Wolters Kluwer/Lippincott Williams \& Wilkins; 2008

37. Schmittgen TD, Livak KJ. Analyzing real-time PCR data by the comparative C (T) method. Nat Protoc. 2008;3(6):1101-8.

38. Carbon S, Ireland A, Mungall CJ, Shu S, Marshall B, Lewis S. AmiGO: online access to ontology and annotation data. Bioinformatics. 2009;25(2):288-9. doi:10.1093/bioinformatics/btn615.

39. Boyle El, Weng S, Gollub J, Jin H, Botstein D, Cherry JM, et al. GO: TermFinder - open source software for accessing Gene Ontology information and finding significantly enriched Gene Ontology terms associated with a list of genes. Bioinformatics. 2004;20(18):3710-5. doi:10.1093/bioinformatics/bth456.

40. Gonçalves C. The Cancer Genome Atlas (TCGA). http://cancergenome.nih.gov/. Accessed 2014

\section{Submit your next manuscript to BioMed Central and take full advantage of:}

- Convenient online submission

- Thorough peer review

- No space constraints or color figure charges

- Immediate publication on acceptance

- Inclusion in PubMed, CAS, Scopus and Google Scholar

- Research which is freely available for redistribution 